\title{
Rab27B-Mediated Metabolic Reprogramming Induces Secretome Acidification and Chemoresistance in Breast Cancer Cells
}

\author{
Original Research Article
}

\author{
An Hendrix ${ }^{1,2, *}$, Carla Ciccone ${ }^{3}$, Christian Gespach ${ }^{4}$, Marc Bracke ${ }^{1}$, \\ Olivier De Wever ${ }^{1}$ and Wendy Westbroek ${ }^{3}$ \\ 1 Laboratory of Experimental Cancer Research, Department of Radiation Oncology and Experimental Cancer Research, Belgium \\ 2 Department of Medical Oncology, Ghent University Hospital, Belgium \\ 3 Medical Genetics Branch, National Human Genome Research Institute, USA \\ 4 INSERM U673 Molecular and Clinical Oncology of Solid Tumors and INSERM U938, \\ Université Pierre et Marie Curie-Paris, Faculté de Médecine, France \\ * Corresponding author E-mail: an.hendrix@ugent.be
}

(C) 2013 Hendrix et al; ;icensee InTech. This is an open access article distributed under the terms of the Creative Commons Attribution License (http://creativecommons.org/licenses/by/3.0), which permits unrestricted use, distribution, and reproduction in any medium, provided the original work is properly cited.

Abstract The secretory Rab27B small GTPase promotes invasive growth, tumourigenicity and metastasis in oestrogen receptor (ER)-positive human breast cancer cells. Coherently, increased Rab27B expression in breast cancer patients is associated with a poor prognosis. In the present study, bio-energetic profiling revealed that oxidative phosphorylation is significantly reduced in ERpositive breast cancer cells engineered to overexpress Rab27B levels as observed in invasive clinical primary breast cancer. Rab27B-induced metabolic reprogramming to aerobic glycolysis was further evidenced by increased extracellular acidification followed by cathepsin B activation and doxorubicin resistance. Transient silencing of Rab27B and stable transfection of Rab27A, and Rab27B mutants in ER-positive breast cancer cells confirmed that this response was Rab27B-specific and dependent upon Rab27B-GTP activation and vesicle membrane attachment through the C-terminal geranylgeranyl group of this small GTPase. Rab27B-driven extracellular acidification is required and is sufficient to induce filopodia-like morphological changes, primarily involved in the process of cancer cell invasion. Our data demonstrate that a Rab27B-dependent switch from oxidative phosphorylation towards aerobic glycolysis in ERpositive breast cancer cells is accompanied by acidification of the tumour environment.

Keywords Rab GTPase, Aerobic Glycolysis, Filopodia

\section{Introduction}

Metastatic cancer is strongly dependent on an adapted metabolism to fulfil the bioenergetic requirements for uncontrolled invasive growth [1]. Proliferating cells are in much greater need of reduced carbon and nitrogen, as well as cytosolic NADPH for reductive biosynthetic reactions [2]. This metabolic phenotype is characterized 
by a shift from oxidative phosphorylation (OXPHOS) towards aerobic glycolysis supplying nucleotides, proteins and lipids for intense and constitutive macromolecular synthesis. Glycolysis first requires the conversion of glucose to pyruvate and eventually to the waste product lactic acid. Glycolysis is inhibited by oxygen consumption, which allows mitochondria to oxidize pyruvate to $\mathrm{CO}_{2}$ and $\mathrm{H}_{2} \mathrm{O}$. Conversion of glucose to lactic acid in the presence of oxygen, but without its consumption, is known as the Warburg effect. Aerobic glycolysis is detected clinically with positron emission tomography to measure the increased uptake of the glucose analogue tracer ${ }^{18}$ fluorodeoxyglucose in many cancers [3].

Altered metabolism in cancer cells is characterized by the production and excretion of lactate, resulting in intratumoural acidification [4]. Typically, the extracellular $\mathrm{pH}$ ( $\mathrm{pHe}$ ) in tumours is heterogeneous with a range of $6.44<\mathrm{pHe}<6.79$ [5]. Low $\mathrm{pH}$ promotes excretion of numerous proteolytic enzymes that are involved in tissue remodelling. Cathepsins are well described as $\mathrm{pH}$ regulated membrane remodelling enzymes with increased secretion and activity in tumours [6]. Acidic pHe contributes to increased exosome release and paracrine exosome uptake [7]. These small membrane vesicles contain numerous proteins, lipids and even nucleic acids and can travel to distant tissues, which allows them to influence multiple aspects of tumour behaviour, including local invasive growth and distant metastasis [8]. Acidity has been shown to play an important role during resistance to chemotherapy by impairing the uptake of weakly basic anti-cancer drugs. For example, doxorubicin (DOX), a well-established drug for breast cancer treatment, is a weak base (pKa between 7.5 and 9.5) that is protonated in the acidic tumour environment and consequently displays decreased cellular uptake [9].

The small GTPase Rab27B regulates the transport and exocytosis of multiple organelles, including multivesicular endosomes (MVEs), resulting in the release of exosomes [10, 11]. Rab27B-mediated derailed exocytosis releases pro-invasive growth regulators into the tumour environment for extracellular matrix degradation. In addition to the reorganization of the actin cytoskeleton and invasion, Rab27B also induces cell cycle progression and proliferation $[8,12]$. These observations prompted us to investigate the effect of Rab27B small GTPase on the bio-energetic profile of breast cancer cells.

\section{Materials and Methods}

\subsection{Cell culture}

We used previously described cell lines that stably express GFP or GFP-Rab fusion proteins: MCF-7 (or
T47D) GFP (GFP control protein), MCF-7 (or T47D) GFPRab27B (Rab27B wild type protein), MCF-7 GFP-Rab27A (Rab27A wild type protein), MCF-7 GFP-Rab27B Q78L (constitutively active mutant protein), MCF-7 GFPRab27B T23N or N133I (inactive mutant proteins defective in GTP binding), MCF-7 GFP-Rab27B GER (geranylgeranylation mutant protein defective in membrane anchoring) [12]. The ER-positive human breast cancer cell lines were maintained in a culture medium (DMEM supplemented with 10\% FBS (serum), 100U/ml penicillin and $100 \mu \mathrm{g} / \mathrm{ml}$ streptomycin) (Invitrogen, Merelbeke, Belgium). To prepare a serum-free conditioned medium (CM), $2 \times 10^{7}$ cells per flask of each cell type were washed three times and incubated for 24 hours at $37^{\circ} \mathrm{C}$ with $15 \mathrm{~mL}$ serum-free culture medium. The medium was harvested, centrifuged at $1250 \mathrm{~g}$ for five minutes at $4^{\circ} \mathrm{C}$ and passed through a $0.22 \mu \mathrm{m}$ filter. $\mathrm{CM}$ was $30 \times$ concentrated at $4^{\circ} \mathrm{C}$ in Centriprep tubes YM-10 (Millipore, Billerica, MA). We transiently expressed Rab27B-specific HP guaranteed siRNA's and a scrambled RNAi negative control into MCF-7 GFP-Rab27B cells according to the manufacturer's guidelines. siRNAs were purchased from Qiagen (Venlo, Netherlands): siRab27B-1 Target 5' AAA CGT GTG GTT TAT AAT GCA 3' and siRab27B-2 Target 5' TAG GAA TAG ACT TTC GGG AAA 3'. Cell morphology and intracellular GFP-Rab27B vesicle distribution were studied using phase contrast and laser scanning confocal microscopy (Zeiss 510 META, Carl Zeiss, Micro-imaging Inc., Heidelberg, Germany) respectively.

\subsection{Bioenergetic profiling}

The XF Extracellular Flux Analyser (Seahorse Bioscience, Billerica, MA) is a 24-well instrument that continuously measures the uptake and excretion of metabolic end products. The XF analyser contains a sensor cartridge, embedded with 24 pairs of fluorescent biosensors $\left(\mathrm{O}_{2}\right.$ and $\mathrm{pH}$ ), which monitor substrate concentration changes by a fibre optic waveguide. The specialized plates allow for the measure of oxygen consumption over time. Cells were seeded at a density of 15,000 in XF24 cell culture microplates (Seahorse Bioscience) in a culture medium and incubated at $37^{\circ} \mathrm{C}$ in $10 \% \mathrm{CO}_{2}$ for 24 hours. The assay is initiated by aspirating the culture medium, replacing it with $1 \mathrm{ml}$ of pre-warmed $\left(37^{\circ} \mathrm{C}\right)$ assay medium (DMEM without bicarbonate but containing sodium pyruvate, GlutaMax-1 and $10 \mathrm{mM}$ glucose, $\mathrm{pH}=7.4$ ) (Invitrogen), to wash the cells. After washing, almost all of the media is removed and an additional $625 \mu \mathrm{l}$ of assay media are added. The plate is incubated at $37^{\circ} \mathrm{C}$ in a non- $\mathrm{CO}_{2}$ incubator for 30 minutes. The calibration plate (Seahorse Bioscience) is prepared one day in advance. The probes on the plate are immersed in calibrate solution (Seahorse Bioscience) for a minimum of 24 hours prior to the experiment and placed at $37^{\circ} \mathrm{C}$ in a non- $\mathrm{CO}_{2}$ incubator. 
On the day of the experiment, the treatments are placed in the ports of the calibration plate. The three treatments, all from Sigma-Aldrich, used in this experiment are FCCP $(200 \mathrm{nM})$, oligomycin $(1 \mu \mathrm{M})$ and rotenone $(1 \mu \mathrm{M})$. Two rows of the plate were injected with all three drugs, while the remaining two rows were injected with assay media only to serve as control wells. The three treatments and media were loaded into the appropriate ports in a volume of $75 \mu \mathrm{l}$. Three measurements were taken as initial basal measurements followed by three measurements after each injection. The wells are mixed for two minutes, there is a three minute wait time and then the wells are measured over a two minute time frame. Oxygen consumption rates $(\mathrm{OCR})$ are reported in $\mathrm{pmol} / \mathrm{min}$ over the time course of the experiment.

\subsection{Measurement of extracellular medium $\mathrm{pH}(\mathrm{pHe})$}

The measurement of $\mathrm{pHe}$ is initiated by aspirating the culture medium of exponential growing cells and washing with a pre-warmed $\left(37^{\circ} \mathrm{C}\right)$ assay medium (DMEM without bicarbonate but containing sodium pyruvate, GlutaMax-1 and $10 \mathrm{mM}$ glucose, $\mathrm{pH}=7.4$ ) (Invitrogen). After washing, additional assay media is added and the cultures are incubated at $37^{\circ} \mathrm{C}$ in a non$\mathrm{CO}_{2}$ incubator. 24 hours later the medium is analysed using a calibrated $\mathrm{pH}$ meter.

\subsection{Western blot analysis}

For western blot analysis, MCF-7 cells $\left(1-10 \times 10^{6}\right)$ were harvested in Laemmli lysis buffer $(0.125 \mathrm{M}$ Tris- $\mathrm{HCl}[\mathrm{pH}$ 6.8 ], $10 \%$ glycerol, $2.3 \%$ sodium dodecyl sulphate [SDS]). Cell lysates $(25 \mu \mathrm{g})$ and $\mathrm{CM}(20 \mu \mathrm{L})$ were suspended in $10 \mu \mathrm{L}$ reducing sample buffer $(1 \mathrm{M}$ Tris- $\mathrm{HCl}$ [pH 6.8], 30\% glycerol, $6 \%$ SDS, 3\% b-mercaptoethanol, $0.005 \%$ bromophenol blue) and boiled for five minutes at $95^{\circ} \mathrm{C}$. Samples were run on NuPage $4 \%-20 \%$ Bis-Tris gradient gels (Invitrogen), transferred to polyvinylidene fluoride membranes, blocked in 5\% non-fat milk in phosphatebuffered saline (PBS) with $0.5 \%$ Tween-20 and stained using rabbit polyclonal anti-cathepsin B (Santa Cruz. Biotechnology, Santa Cruz, CA), mouse monoclonal antiPARP (BD Pharmigen, Belgium) and mouse monoclonal anti-tubulin (Sigma-Aldrich, St Louis, MO) antibodies.

\section{Results}

\subsection{Rab27B attenuates OXPHOS capacity}

To investigate the impact of Rab27B on energy metabolism pathways we tested pharmacologic modulators interfering with the mitochondrial respiratory pathway in the Seahorse XF24 Extracellular Flux Analyser [13]. We engineered MCF-7 breast cancer cells stably overexpressing Rab27B similarly to levels found in invasive breast tumours [12]. Treatments were performed with the uncoupler FCCP (carbonyl cyanide p(trifluoromethoxy) phenylhydrazone) (30, 40 and 50 minutes), the complex $\mathrm{V}$ inhibitor oligomycin (60, 70 and 80 minutes) and the complex I inhibitor rotenone (90 and 100 minutes). After each drug treatment, we observed that the oxygen consumption rate (OCR) was significantly lower in GFP-Rab27B transfected MCF-7 cells compared to MCF-7 GFP cells, suggesting that Rab27B decreased OXPHOS activity and increased dependency on aerobic glycolysis (Figure 1). This is consistent with our previous finding that Rab27B stimulates invasive tumour growth in a xenograft mouse model, a high-energy dependent process [12].

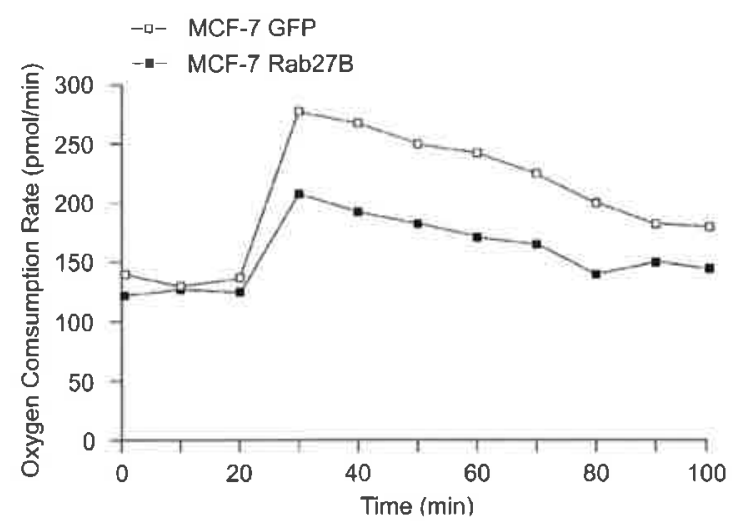

Figure 1. Reduction of oxidative phosphorylation by Rab27B in human breast cancer cells MCF-7. Real-time analysis of the oxygen consumption rate (OCR) by XF Extracellular flux Analyzer in MCF-7 GFP and GFP-Rab27B transfected cells. Timepoints (minutes) one, 10 and 20 represent baseline measurements before drug treatment; 30,40 and 50: OCR measurements after carbonyl cyanide $\mathrm{p}$-(trifluoromethoxy) phenylhydrazone (FCCP) treatment; 60, 70 and 80: OCR after oligomycin addition; 90 and 100: measurements after rotenone treatment. OCR measurements are the mean of three independent experiments.

\subsection{Rab27B promotes acidic extracellular medium $\mathrm{pHe}$}

In exponentially growing cell cultures, Rab27B reduced the extracellular medium pHe of MCF-7 cells by 0.64 units after 24 hours, from 7.40 to 6.76 (Figure 2a). In contrast, $\mathrm{pHe}$ of control vector transfected MCF-7 cells was 7.14. Importantly, Rab27A overexpression had no significant effect on pHe. In addition, MCF-7 cells overexpressing Rab27B defective in GTP-binding (i.e., inactive Rab27B protein, N133I) or membrane anchoring (i.e., no geranylgeranylation of the protein, GER) were both ineffective in regulating $\mathrm{pHe}$. Transient targeting by a single siRNA depleted Rab27B protein by $70-80 \%$ and resulted in loss of extracellular acidification (Figure $2 b$ ). Similar observations were made when pHe was measured in another model, the T47D breast cancer cells overexpressing Rab27B (data not shown). 


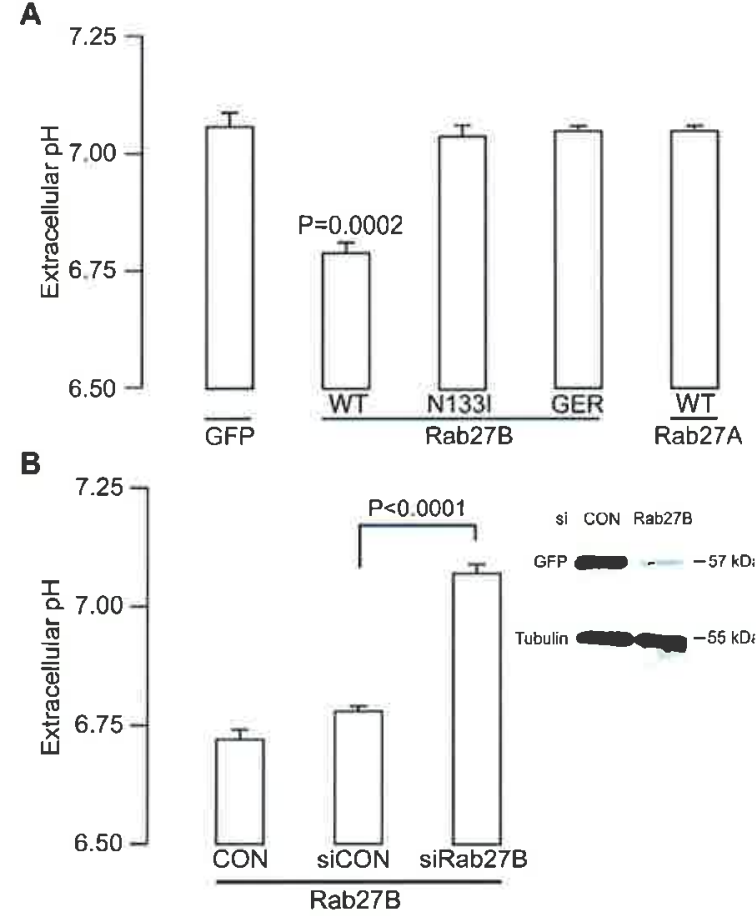

Figure 2. Role of Rab27B in the extracellular acidification process. (A) pHe measurement of the culture medium prepared from MCF-7 cells stably transfected with GFP-Rab27B (WT), GFP-Rab27B mutants defective in GTP-binding (N133I) or vesicle membrane anchoring (GER) N133I and GER or GFPRab27A (WT) were compared with pHe values recorded from MCF-7 GFP cells. Cells were cultured for 24 hours during the exponential growth after assay medium refreshment. (B) Control MCF-7 GFP-Rab27B cells (CON) and MCF-7 GFP-Rab27B cells transfected with scrambled siRNA (siCON) or Rab27B siRNA, were cultured under exponential growth conditions. After 72 hours, the culture medium was refreshed and $\mathrm{pHe}$ was measured 24 hours later. Inset: Silencing of Rab27B after 96 hours of Rab27B siRNA or siCON transfection in MCF-7 GFPRab27B cells assessed by western blot. All pHe data are expressed as the mean \pm s.e.m. of three independent experiments performed in triplicate. P-values are calculated using the twosided Student's t-test following D'Agostino-Pearson testing for normal distribution. Statistically significant P-values are indicated.

\subsection{Functional consequences of Rab27B-induced acidification}

MCF-7 cells secrete cathepsin B, a cysteine protease that is activated by acidic pHe. We therefore analysed cathepsin B activation in the conditioned media of MCF7 GFP and GFP-Rab27B cells. Western blot analysis showed that the cathepsin B protein is exclusively activated in the extracellular milieu of MCF-7 GFPRab27B cells (Figure 3a). However, cathepsin B is not trafficked by secretory Rab27B vesicles: 1) mass spectrometry analysis of the Rab27B vesicle content did not reveal cathepsin B protein [12] and 2) confocal microscopy revealed that cathepsin B did not co-localize with Rab27B-positive vesicles (data not shown). In addition, overexpression of Rab27B in MCF-7 cells decreased 3.3-fold the potency of the cytotoxic agent doxorubicin (DOX) $(\mathrm{IC} 50=1.56 \mu \mathrm{M})$, compared to MCF7 GFP cells ( $\mathrm{IC} 50=0.47 \mu \mathrm{M}, \mathrm{P}<0.001$ ). This was further evidenced by absence of the $85 \mathrm{kDa}$ cleaved active poly(ADP-ribose)polymerase-1 (PARP) fragment involved in cell death pathways (Figure $3 b$ ).

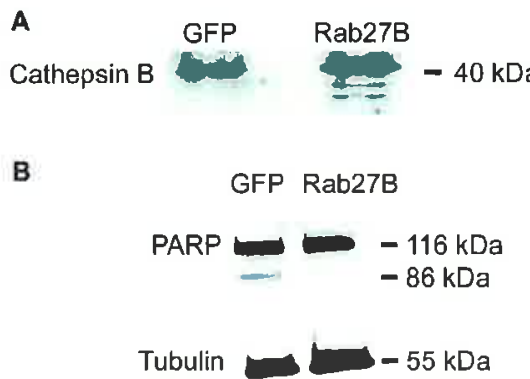

Figure 3. Impact of Rab27B-mediated extracellular acidification on cathepsin B activation and PARP cleavage. (A) Western blot analysis of cathepsin $\mathrm{B}$ in the conditioned culture media of MCF7 GFP and MCF-7 GFP-Rab27B cells. Pro-cathepsin B has a molecular weight of $40 \mathrm{kDa}$, while maturated cathepsin $\mathrm{B}$ is characterized by protein bands at 25 and $30 \mathrm{kDa}$. (B) Expression and cleavage of the apoptosis-related protein PARP $(85 \mathrm{kDa})$ analyzed by western blot. Tubulin $(55 \mathrm{kDa})$ was used as loading control.

\subsection{Rab27B induced acidic $p H e$ promotes filopodia formation}

Next, we analysed the impact of induced and reversed acidification on the morphology of MCF-7 cells under control and Rab27B conditions. pHe was experimentally adjusted to physiological and acidic pHe (respectively 7.2 and 6.5) for 24 hours in MCF-7 GFP and GFP-Rab27B cell cultures. In this pHe range, we observed that experimental acidification of the culture medium of MCF7 GFP cells for 24 hours did not induce any morphological changes (Figure 4a). In contrast, MCF-7 GFP-Rab27B cells displayed more filopodia extrusions at acidic $\mathrm{pHe}$ as compared to cells maintained at physiological pHe (Figure 4a). These filopodia often contained Rab27B-positive vesicles at their distal ends (Figure 4c). This was not the case in MCF-7 GFP cells cultured at these two experimentally adjusted pHe conditions. In a next step we compared the morphological impact of acidified culture medium induced by Rab27B-dependent mechanisms (Figure $4 \mathrm{~b}$ ) with experimental and direct acidification of the culture medium prepared from MCF-7 GFP cells (Figure 4a, lower left panel). Only transfer of culture medium from MCF-7 GFP-Rab27B cells induced the formation of filopodia protrusions in MCF-7 GFP acceptor cells (Figure 4b). 

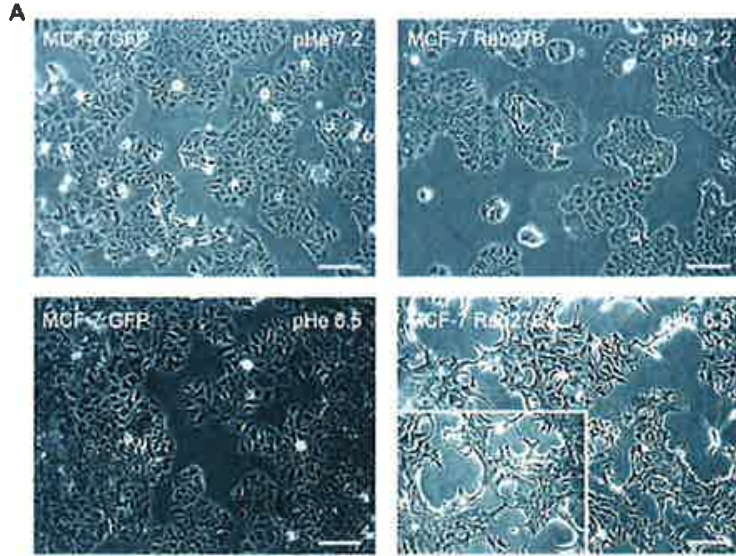

B
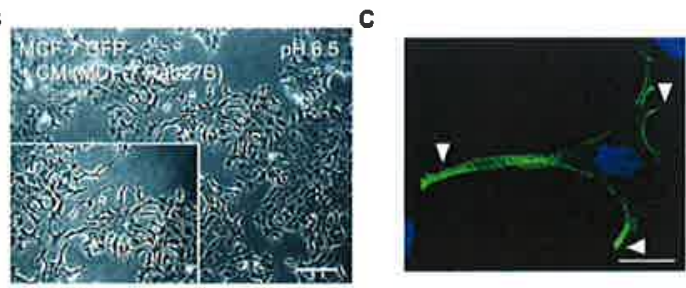

Figure 4. Influence of manipulating directly extracellular $\mathrm{pH}$ at physiological and acidic values (pH 7.2 versus 6.5 ) on the morphology by phase-contrast microscopy of control and Rab27B-transfected MCF-7 cells. (A) MCF-7 GFP (left) and GFPRab27B cells (right) were cultured at pHe 7.2 (upper panels) or pHe 6.5 (lower panels). Filopodia formation was induced only in Rab27B-transfected cells maintained at acidic pHe. Scale bar: $100 \mu \mathrm{m}$. (B) Control MCF-7 GFP cells treated with the conditioned media prepared from MCF-7 GFP-Rab27B cell cultures maintained at acidic $\mathrm{pHe}$ induced the production filopodia extensions. Scale bar: $100 \mu \mathrm{m}$. (C) Representative laser scarning confocal image of GFP-Rab27B positive vesicle distribution. Arrowheads indicate GFP-Rab27B vesicle accumulation in filopodia protrusions. Scale bar: $20 \mu \mathrm{m}$.

\section{Discussion}

Our data suggest that Rab27B up-regulation in ERpositive breast cancer cells induces a switch from oxidative phosphorylation towards aerobic glycolysis, to sustain the energy demands required for invasive tumour growth, which is accompanied by acidification of the tumour environment. This metabolic reprogramming is Rab27B-specific and exclusively requires GTP-binding and geranylgeranyl-dependent membrane targeting.

The metabolism of cancer cells is reprogrammed to meet the challenges of macromolecular synthesis associated with cell growth and proliferation [2]. This altered metabolism can be related to growth factor signalling since activated PI3K/Akt signalling downstream of receptor tyrosine kinase activation leads to enhanced glucose uptake and glycolysis [14, 15]. How can altered expression of the small GTPase Rab27B contribute to a metabolic shift? Rab27B transports MVEs towards the plasma membrane and fusion results in the release of exosomes [11]. We previously demonstrated that Rab27Bassociated vesicles release HSP90 $\alpha$, recently identified as an exosome surface protein, in the tumour environment $[12,16]$. Extracellular HSP90 $\alpha$ activates a cassette of proteins that function collectively in invasive growth [8]. Extracellular HSP90 $\alpha$ interacts with the extracellular domain of HER-2 tyrosine kinase receptor in breast cancer cells, which is necessary for receptor activation and heterodimerization with ligand-activated HER-3. This will in turn mediate signal transduction pathways via MAPK and PI3K-Akt, known to play key roles in cellular proliferation, differentiation, migration and metabolic reprogramming [14, 17]. Thus, Rab27B-induced HSP90 $\alpha$ release can contribute to metabolic reprogramming required to sustain invasive growth. Other members of the Ras superfamily, such as Rho GTPases, have been linked to cellular metabolism through glutaminase activity and glutamine dependence. siRNA knockdown or pharmacological inhibition of glutaminase inhibits Rho GTPase induced transformation and proliferation [18]. Rab25, a small GTPase involved in endosomal recycling, which is genomically amplified in multiple cancers, is a key regulator of cellular bioenergetics. RAB25 induced an Akt-dependent increase in glucose uptake and glycogen storage which allows maintenance of ATP levels during bioenergetic stress [19].

In addition to acidic $\mathrm{pHe}$, a side effect of aerobic glycolysis, we have shown here that the secretome of MCF-7 GFP-Rab27B cells is required to induce filopodia formation. This suggests that the Rab27B secretome contains putative positive regulators of the small GTPase Rho involved in filopodia formation [20]. These results also indicate a paracrine diffusion of the filopodia phenotype from Rab27B transfected breast cancer cells to the control cells, potentially by the means of exosomes. It has been demonstrated that addition of exosomes containing notch ligand Delta-like four confer a tip cell phenotype on endothelial cells characterized by filopdia formation [21]. The relocalization of Rab27B-associated vesicles to the filopodia protrusions may facilitate exocytosis at sites of active migration and matrix remodelling.. Similar observations were published by Glunde et al. demonstrating that secretory lysosome distribution in breast cancer cells alters at acidic pHe [22]. Human melanoma cells exhibit more lamellipodia and stronger adhesion at lower $\mathrm{pHe}$, and displayed enhanced invasion and increased secretion of proteases and proangiogenic factors in response to extracellular acidification. Also, in human breast cancer cells, the number and length of filopodia has been shown to increase at acidic $\mathrm{pH}$ [22]. In conclusion, our data demonstrate a Rab27B-dependent switch from oxidative phosphorylation towards aerobic glycolysis in ERpositive breast cancer cells to sustain invasive growth in acidified environments. 


\section{Conflict of interest}

The authors declare no conflict of interest.

\section{Acknowledgements}

This work was in part supported by the Intramural Research Program of the National Human Genome Research Institute (NIH, Bethesda, Maryland), INSERM Research Center U938 and Paris VI University UPMC, Spearhead Oncology of Ghent University Hospital, a postdoctoral grant (ODW and $\mathrm{AH}$ ) from Fund for Scientific Research-Flanders and Special Research Fund Ghent University.

\section{References}

[1] Vander Heiden MG, Cantley LC, Thompson CB: Understanding the Warburg effect: the metabolic requirements of cell proliferation. Science 2009, 324(5930): 1029-1033.

[2] Ward PS, Thompson CB: Metabolic reprogramming a cancer hallmark even warburg did not anticipate. Cancer Cell 2012, 21(3):297-308.

[3] Gatenby RA, Gillies RJ: Why do cancers have high aerobic glycolysis? Nat Rev Cancer 2004, 4(11):891899.

[4] Fais S, De Milito A, You H, Qin W: Targeting vacuolar $\mathrm{H}+$-ATPases as a new strategy against cancer. Cancer Res 2007, 67(22):10627-10630.

[5] van Sluis $R$, Bhujwalla ZM, Raghunand $N$, Ballesteros P, Alvarez J, Cerdan S, Galons JP, Gillies RJ: In vivo imaging of extracellular $\mathrm{pH}$ using $1 \mathrm{H}$ MRSI. Magn Reson Med 1999, 41(4):743-750.

[6] Chapman HA, Riese RJ, Shi GP: Emerging roles for cysteine proteases in human biology. Annu Rev Physiol 1997, 59:63-88.

[7] Parolini I, Federici C, Raggi C, Lugini L, Palleschi S, De Milito A, Coscia C, Iessi E, Logozzi M, Molinari A et al.: Microenvironmental $\mathrm{pH}$ is a key factor for exosome traffic in tumor cells. J Biol Chem 2009, 284(49):34211-34222.

[8] Hendrix A, Westbroek W, Bracke M, De Wever O: An ex(o)citing machinery for invasive tumor growth. Cancer Res 2010, 70(23):9533-9537.

[9] Alabaster O, Woods T, Ortiz-Sanchez $V_{r}$ Jahangeer S: Influence of microenvironmental $\mathrm{pH}$ on adriamycin resistance. Cancer Res 1989, 49:5638-5643.

[10] Hendrix A, Hume AN: Exosome signaling in mammary gland development and cancer. Int $J$ Dev Biol 2011, 55(7-9):879-887.

[11] Ostrowski M, Carmo NB, Krumeich S, Fanget I, Raposo G, Savina A, Moita CF, Schauer K, Hume AN, Freitas RP, et al.: Rab27a and Rab27b control different steps of the exosome secretion pathway. Nat Cell Biol 2010, 12(1):19-30.
[12] Hendrix A, Maynard D, Pauwels P, Braems G, Denys $\mathrm{H}$, Van den Broecke R, Lambert J, Van Belle S, Cocquyt V, Gespach $\mathrm{C}$ et al: Effect of the secretory small GTPase Rab27B on breast cancer growth, invasion and metastasis. I Natl Cancer Inst 2010, 102(12):866-880

[13] Wu M, Neilson A, Swift AL, Moran R, Tamagnine J, Parslow D, Armistead S, Lemire K, Orrell J, Teich J et al: : Multiparameter metabolic analysis reveals a close link between attenuated mitochondrial bioenergetic function and enhanced glycolysis dependency in human tumor cells. Am J Physiol Cell Physiol 2007, 292(1):125-136.

[14] Buzzai M, Bauer DE, Jones RG, Deberardinis RJ, Hatzivassiliou G, Elstrom RL, Thompson CB: The glucose dependence of Akt-transformed cells can be reversed by pharmacologic activation of fatty acid beta-oxidation. Oncogene 2005, 24(26):4165-4173.

[15] Elstrom RL, Bauer DE, Buzzai M, Karnauskas R, Harris MH, Plas DR, Zhuang H, Cinalli RM, Alavi A, Rudin CM et al.: Akt stimulates aerobic glycolysis in cancer cells. Cancer Res 2004, 64(11):3892-3899.

[16] Epple LM, Griffiths SG, Dechkovskaia AM, Dusto NL, White J, Ouellette RJ, Anchordoquy TJ, Bemis LT, Graner MW: Medulloblastoma exosome proteomics yield functional roles for extracellular vesicles. PLoS One. 2012, 7(7):e42064.

[17] Sidera K, Gaitanou M, Stellas D, Matsas R, Patsavoudi E: A critical role for HSP90 in cancer cell invasion involves interaction with the extracellular domain of HER-2. J Biol Chem 2008, 283(4):2031-2041.

[18] Wang JB, Erickson JW, Fuji R, Ramachandran S, Gao $\mathrm{P}$, Dinavahi $\mathrm{R}$, Wilson KF, Ambrosio AL, Dias SM, Dang CV et al.: Targeting mitochondrial glutaminase activity inhibits oncogenic transformation. Cancer Cell, 18(3):207-219.

[19] Cheng KW, Agarwal R, Mitra S, Lee JS, Carey M, Gray JW, Mills GB: Rab25 increases cellular ATP and glycogen stores protecting cancer cells from bioenergetic stress. EMBO Mol Med, 4(2):125-141.

[20] Hall A: Rho GTPases and the actin cytoskeleton. Science 1998, 279(5350):509-514.

[21] Sheldon H, Heikamp E, Turley H, Dragovic R, Thomas P, Oon CE, Leek R, Edelmann M, Kessler B, Sainson RC, et al. New mechanism for Notch signaling to endothelium at a distance by Delta-like 4 incorporation into exosomes. Blood 2010, 116(13):2385-94.

[22] Glunde K, Guggino SE, Solaiyappan M, Pathak AP, Ichikawa $Y$, Bhujwalla ZM: Extracellular acidification alters lysosomal trafficking in human breast cancer cells. Neoplasia 2003, 5(6):533-545. 